\title{
L'étude du comportement du malade (Illness Behaviour) comme base de la planification de l'éducation à la santé
}

\author{
H. R. M. Müller 1
}

Division de Médecine Sociale et Préventive de l'Université de Bâle

\section{Résumé 1}

Un modèle du comportement du malade doit comprendre les différents facteurs du milieu social, de l'apprentissage social et de la personnalité. Par l'élabora-

\footnotetext{
${ }^{1}$ Le texte intégral en langue allemande paraîtra dans le no 6/1977 de cette Revue.
}

tion de beaucoup de projets de recherce on a développé des concepts qui peuvent servir de «variables explicatives». Ce modèle, qui saisit tous les éléments importants du comportement du malade, sert de cadre à une recherche épidémiologique chez des enfants d'âge scolaire. Les résultats serviront de base à la planification de programme dans l'éducation à la santé. 